\title{
Right orders and amalgamation for lattice-ordered groups
}

\author{
V. V. Bludov and A. M. W. Glass
}

May 13, 2009

Dedicated to W. Charles Holland on his $75^{\text {th }}$ birthday, with gratitude for his research.

\begin{abstract}
Let $H_{i}$ be a sublattice subgroup of a lattice-ordered group $G_{i}$ $(i=1,2)$. Suppose that $H_{1}$ and $H_{2}$ are isomorphic as latticeordered groups, say by $\varphi$. In general, there is no lattice-ordered group in which $G_{1}$ and $G_{2}$ can be embedded (as lattice-ordered groups) so that the embeddings agree on the images of $H_{1}$ and $H_{1}^{\varphi}$. In this article we prove that the group free product of $G_{1}$ and $G_{2}$ amalgamating $H_{1}$ and $H_{1}^{\varphi}$ is right orderable and so embeddable (as a group) in a lattice-orderable group. To obtain this, we use our necessary and sufficient conditions for the free product of right-ordered groups with amalgamated subgroup to be right orderable [1]. We also provide new limiting examples to show that amalgamation can fail in the category of lattice-ordered groups even when the amalgamating sublattice subgroups are convex and normal ( $\ell$-ideals) and solve Problem 1.42 of [6].
\end{abstract}

AMS Classification: 06F15, 20F60, 20B27, 20F10. 


\section{Introduction}

A partially ordered group (or p.o. group, for short) is a group with a partial order $\leq$ such that $a x b \leq a y b$ whenever $x \leq y$. A special case is provided when the partial order is a lattice. A lattice-ordered group $G$ is a group and a lattice such that

$$
a(x \vee y) b=a x b \vee a y b \quad \text { and } \quad a(x \wedge y) b=a x b \wedge a y b
$$

for all $a, b, x, y \in G$. We will frequently use the abbreviation $\ell$-group for lattice-ordered group. We will write $\ell$-subgroup as a shorthand for a sublattice subgroup, $\ell$-homomorphism for a map that is both a group and a lattice homomorphism, etc. Lattice operations $\vee$ and $\wedge$ on an $\ell$-group $G$ induce a partial order $\leq$ on $G: g \leq h$ if and only if $g \vee h=h$ (or equivalently $g \wedge h=g$ ). We say that $g_{1}$ and $g_{2}$ are orthogonal if $g_{1} \wedge g_{2}=1$. A normal convex $\ell$-subgroup of an $\ell$-group is called an $\ell$ ideal. An $\ell$-ideal $N$ of an $\ell$-group $G$ is called prime if the $\ell$-group $G / N$ (with the naturally induced lattice order) is totally ordered. Thus an $\ell$-ideal $N$ of an $\ell$-group $G$ is prime if and only if for any pair $g_{1}, g_{2}$ of orthogonal elements of $G$, at least one of $g_{1}$ and $g_{2}$ belongs to $N$.

There are two especially important ways to form new $\ell$-groups from old. If $G_{1}$ and $G_{2}$ are $\ell$-groups, we can partially order their direct product $G_{1} \times G_{2}$ by: $\left(g_{1}, g_{2}\right) \geq 1$ if and only if $g_{i} \geq 1(i=1,2)$. This is called the cardinal product of $G_{1}$ and $G_{2}$; it is an $\ell$-group. Note that if $f_{i} \in G_{i}$ with $f_{i} \geq 1(i=1,2)$, then the images of $f_{1}$ and $f_{2}$ in the cardinal product of $G_{1}$ and $G_{2}$ are orthogonal. Let $K$ be an o-group (i.e., an $\ell$-group with the order total). If $G$ is an $\ell$-group and $K$ acts on $G$ as a group of $\ell$-automorphisms, then the splitting extension of $G$ by $K$ is an $\ell$-group, where $1 \leq(g, k)$ if and only if $k>1$ or both $k=1$ and $g \geq 1$. We write $G \overleftarrow{\rtimes} K$ for this $\ell$-group and call it the lexicographic split extension of $G$ by $K$.

A right-ordered group $G$ is a group with a total order $\leq$ such that $x g \leq y g$ whenever $x \leq y$ in $G$. Every right-ordered group can be (group) embedded in a lattice-ordered group; and every lattice-ordering on a group is the intersection of all the right orders on the group that extend the original lattice ordering (see [2], Examples 1.3.19 and 1.3.20). This close relationship between right orders and lattice orders is at the heart of this article. 
Given groups $G_{1}$ and $G_{2}$ with isomorphic subgroups $H_{1}$ and $H_{2}$ respectively (say $\varphi: H_{1} \cong H_{2}$ ), the free product of $G_{1}$ and $G_{2}$ with $H_{1}$ and $H_{2}$ amalgamated by $\varphi$ is the quotient of $G_{1} * G_{2}$ by the normal subgroup generated by $\left\{h_{1}^{\varphi} h_{1}^{-1} \mid h_{1} \in H_{1}\right\}$. We denote it by $G_{1} * G_{2}\left(H_{1} \stackrel{\varphi}{\cong} H_{2}\right)$. The main result is that the group $G_{1} * G_{2}\left(H_{1} \stackrel{\varphi}{\cong} H_{2}\right)$ with natural maps from $G_{1}$ and $G_{2}$ is an amalgam; i.e., it is the "free-est" such generated by the union of the images of $G_{1}$ and $G_{2}$ where the natural maps are embeddings.

In [1], we determined necessary and sufficient conditions for a free product of groups with amalgamated subgroups to be right orderable. Let $G$ be a right orderable group and $\mathcal{R}$ be a non-empty family of right orders on $G$. $\mathcal{R}$ is said to be a normal family if $\leq \in \mathcal{R}$ and $g \in G$ imply that $\leq^{g} \in \mathcal{R}$, where $a \leq^{g} b$ if and only if $g a \leq g b$. Let $G_{i}$ be a right orderable group and $\mathcal{R}_{i}$ be a family of right orders on $G_{i}(i=1,2)$. If $H_{i}$ is a subgroup of $G_{i}(i=1,2)$ with $H_{1}$ and $H_{2}$ isomorphic (say $\left.\varphi: H_{1} \cong H_{2}\right)$, then we say that $\left(\mathcal{R}_{1}, \mathcal{R}_{2}\right)$ is compatible for $\varphi$ if

$$
\left(\forall \leq_{1} \in \mathcal{R}_{1}\right)\left(\exists \leq_{2} \in \mathcal{R}_{2}\right)\left(\forall h_{1} \in H_{1}\right)\left(1 \leq_{1} h_{1} \Longrightarrow 1 \leq_{2} h_{1}^{\varphi}\right),
$$

and

$$
\left(\forall \leq_{2} \in \mathcal{R}_{2}\right)\left(\exists \leq_{1} \in \mathcal{R}_{1}\right)\left(\forall h_{2} \in H_{2}\right)\left(1 \leq_{2} h_{2} \Longrightarrow 1 \leq_{1} h_{2}^{\varphi^{-1}}\right) .
$$

We proved

Theorem 1 ([1], Theorem A). Let $H_{i}$ be a subgroup of a right-orderable group $G_{i}(i=1,2)$. Assume that $\varphi: H_{1} \cong H_{2}$ is an isomorphism. Then $L:=G_{1} * G_{2}\left(H_{1} \stackrel{\varphi}{\cong} H_{2}\right)$ is right orderable if and only if there is a normal family $\mathcal{R}_{i}$ of right orders on $G_{i}(i=1,2)$ such that $\left(\mathcal{R}_{1}, \mathcal{R}_{2}\right)$ is compatible for $\varphi$. Moreover, the right orders $\leq_{i}$ on $G_{i}(i=1,2)$ extend to a right order on $L$ if and only if $\left(\leq_{1}, \leq_{2}\right)$ is compatible with $\varphi$ and $\leq_{i}$ is contained in a normal family $\mathcal{R}_{i}$ of right orders on $G_{i}(i=1,2)$ such that $\left(\mathcal{R}_{1}, \mathcal{R}_{2}\right)$ is compatible for $\varphi$.

We use this result to prove

Theorem A Let $H_{i}$ be a sublattice subgroup of a lattice-ordered group $G_{i}$ $(i=1,2)$. If $\varphi: H_{1} \cong H_{2}$ is an $\ell$-isomorphism, then $G_{1} * G_{2}\left(H_{1} \cong H_{2}\right)$ is right orderable. 
We can also obtain lattice orderable conditions equivalent to the group $G_{1} * G_{2}\left(H_{1} \stackrel{\varphi}{\cong} H_{2}\right)$ being right orderable.

Theorem B Let $G_{1}, G_{2}$ be right-orderable groups with isomorphic subgroups $H_{1} \stackrel{\varphi}{\cong} H_{2}$ respectively. Then $G_{1} * G_{2}\left(H_{1} \stackrel{\varphi}{\cong} H_{2}\right)$ is right orderable if and only if there are lattice-ordered groups $\hat{G}_{i}$, group embeddings $\varepsilon_{i}: G_{i} \rightarrow \hat{G}_{i}(i=1,2)$ and an $\ell$-isomorphism $\hat{\varphi}$ between the sublattice subgroups generated by $H_{1}^{\varepsilon_{1}}$ and $H_{2}^{\varepsilon_{2}}$ such that

$$
h_{1}^{\varphi \varepsilon_{2}}=h_{1}^{\varepsilon_{1} \hat{\varphi}}
$$

for all $h_{1} \in H_{1}$.

Since every right-orderable group can be embedded in a lattice-orderable group, we obtain

Corollary 1.1 Let $H_{i}$ be a sublattice subgroup of a lattice-ordered group $G_{i}(i=1,2)$. If $\varphi: H_{1} \cong H_{2}$ is an $\ell$-isomorphism, then there is a latticeordered group $L$ and group embeddings $\varepsilon_{i}: G_{i} \rightarrow L(i=1,2)$ such that $g_{1}^{\varepsilon_{1}}=g_{2}^{\varepsilon_{2}}$ if and only if $g_{1} \in H_{1}$ and $g_{2}=g_{1}^{\varphi}$.

This is in sharp contrast to the lattice-ordered case which is false in general even when the phrase "and only if" is removed (see [5], or [2], Theorem 7.C). We strengthen this to show that the corollary fails if we replace "group embeddings" by " $\ell$-embeddings" even when both amalgamated subgroups are $\ell$-ideals and one of them is prime (Example 5.2). However, such $\ell$-embeddings are possible in some partial cases that we study in Section 4 (see Propositions $4.1-4.3$ ). This situation (when group embeddings are possible and the right orders extend but no $\ell$ embeddings are possible) allows us to construct a p.o. group giving a negative answer for Problem 1.42 listed in [6] (see Example 6.1).

\section{Notation and Preliminaries}

If $H$ is a subset of a right-ordered or lattice-ordered group $G$, let $\operatorname{Conv}_{G}(H)$ denote the least convex subgroup of $G$ containing $H$. It is the intersection of all convex subgroups of $G$ containing $H$. If $H$ is an $\ell$-subgroup of an $\ell$-group $G$, then $\operatorname{Conv}_{G}(H)=\{g \in G \mid(\exists h \in H)(|g| \leq|h|)\}$, where 
$|x|:=x \vee x^{-1}$. Note that $|x| \geq 1$ with $|x|=1$ if and only if $x=1$ (see [2], Lemma 2.3.8).

If $H$ is subset of an $\ell$-group $G$, we write $\langle H\rangle_{\ell}$ for the least $\ell$-subgroup of $G$ containing $H$.

By Zorn's Lemma, if $G$ is an $\ell$-group and $g \in G \backslash\{1\}$, there is a convex $\ell$-subgroup $C_{g}$ of $G$ that is maximal with respect to not containing $g$. It is called a value of $g$. The set $R\left(C_{g}\right)$ of all right cosets of $C_{g}$ in $G$ inherits a total order from $G$ in the natural way (see [2], Corollary 3.3.7).

Let $(\Omega, \leq)$ be a totally ordered set. Then $\operatorname{Aut}(\Omega, \leq)$ is an $\ell$-group under the lattice ordering given by $\omega(g \vee h):=\max \{\omega g, \omega h\}$ and $\omega(g \wedge$ $h):=\min \{\omega g, \omega h\}$ for all $\omega \in \Omega$.

One of the main tools in the theory of $\ell$-groups is in the same spirit as Cayley's permutation representation, though the proof is far less straightforward.

Theorem 2 (Holland [3]). Every lattice-ordered group $G$ is $\ell$-isomorphic to a sublattice subgroup of $\operatorname{Aut}(\Omega, \leq)$ for some totally ordered set $(\Omega, \leq)$.

We may therefore regard any $\ell$-group $G$ as an $\ell$-subgroup of $\mathrm{A}(\Lambda):=$ $\operatorname{Aut}(\Lambda, \leq)$ for a suitable totally ordered set $\Lambda=\Lambda(G)$.

We first recall some standard notation. For $g \in G \leq \mathrm{A}(\Lambda)$, let

$$
\operatorname{fix}(g)=\{\lambda \in \Lambda \mid \lambda g=\lambda\}, \quad \operatorname{supp}(g)=\{\lambda \in \Lambda \mid \lambda g \neq \lambda\} .
$$

So for all $g \in G$,

$$
\begin{gathered}
\operatorname{fix}(g)=\operatorname{fix}\left(g^{-1}\right), \quad \operatorname{fix}\left(g^{h}\right)=\operatorname{fix}(g) \cdot h, \\
\operatorname{supp}(g)=\operatorname{supp}\left(g^{-1}\right), \quad \operatorname{supp}\left(g^{h}\right)=\operatorname{supp}(g) \cdot h,
\end{gathered}
$$

and

$$
\Lambda=\operatorname{fix}(g) \cup \operatorname{supp}(g) .
$$

We also use the standard notation

$$
g^{+}=g \vee 1, \quad g^{-}=g^{-1} \vee 1 .
$$

Indeed (see [2], Lemma 2.3.8),

$$
g=g^{+}\left(g^{-}\right)^{-1}, \quad|g|=g^{+} \vee g^{-}, \quad g^{+} \wedge g^{-}=1 .
$$


For $g \in G$, let

$$
S_{+}(g):=\operatorname{supp}\left(g^{+}\right), \quad S_{-}(g):=\operatorname{supp}\left(g^{-}\right) .
$$

So

$$
S_{+}(g)=\{\lambda \in \Lambda \mid \lambda g>\lambda\}=S_{-}\left(g^{-1}\right), \quad S_{+}\left(g^{h}\right)=S_{+}(g) \cdot h
$$

and

$$
S_{-}(g)=\{\lambda \in \Lambda \mid \lambda g<\lambda\}=S_{+}\left(g^{-1}\right) \quad S_{-}\left(g^{h}\right)=S_{-}(g) \cdot h .
$$

In any $\ell$-group $G$,

$$
S_{+}(g) \cap S_{+}(h)=\emptyset \quad \Longleftrightarrow \quad g^{+} \wedge h^{+}=1 ;
$$

i.e., if and only if $g^{+}$and $h^{+}$are orthogonal.

Clearly, for each $g \in G$,

$$
\left\{S_{+}(g), \operatorname{fix}(g), S_{-}(g)\right\} \text { is a partition of } \Lambda \text {. }
$$

We use a corollary that is easily deduced from the proof of Theorem 2.

Corollary 2.1 For any lattice-ordered group $G$, there is a totally ordered set $\Lambda$ and $\ell$-embedding of $G$ into $\mathrm{A}(\Lambda)$ satisfying

(I) if $L$ is a proper convex $\ell$-subgroup of $G$, then $\operatorname{supp}(g) \cap \operatorname{fix}(h) \neq \emptyset$ for all $g \in G \backslash L, h \in L$, and

(II) $\operatorname{fix}(g) \neq \emptyset$ for all $g \in G$.

Proof. The proof is a minor modification of Holland's original embedding (see [3] or [2], Theorem 7.A); we use all values of all nonidentity elements of $G$ instead of just one value for each non-identity element. Let $\Gamma(G)$ denote the set of all values of all nontrivial elements $g$ from $G$. Order $\Gamma(G)$ by some totaly order $\preceq$. Totally order $\Omega:=\bigcup\{R(C) \mid C \in \Gamma(G)\}$ by: $C_{1} g \leq C_{2} f$ if and only if $C_{1} \prec C_{2}$ or both $C_{1}=C_{2}$ and $C_{1} g \leq C_{1} f$ in $R\left(C_{1}\right)$. We can $\ell$-embed $G$ in $A(\Omega)$ by $(C f) g=C f g(f, g \in G)$. We adjoin to $\Omega$ an extra point at plus infinity, $\nu$; i.e., $\Lambda=\Omega \cup\{\nu\}$, where $\omega<\nu$ for all $\omega \in \Omega$, and $\nu g=\nu$ for all $g \in G$. Thus property (II) holds. Property (I) also holds because $L$ is contained in some value $C_{g}$ of $g \in G \backslash L$ and $C_{g}$ is a point in $\Lambda$ with the 
desired property: $C_{g} g \neq C_{g}$ and $C_{g} h=C_{g}$ for all $h \in L \subseteq C_{g}$. Thus $C_{g} \in \operatorname{supp}(g) \cap \operatorname{fix}(h) \neq \emptyset$. //

Recall that an ultrafilter on $\Lambda$ is a maximal filter on $\Lambda$. A family of subsets of $\Lambda$ forms an ultrafilter $\mathfrak{U}$ on $\Lambda$ if and only if $\mathfrak{U}$ satisfies the properties:

$(\mathrm{U} 1) \emptyset \notin \mathfrak{U}$

(U2) $S, T \in \mathfrak{U}$ imply $S \cap T \in \mathfrak{U}$;

(U3) if $S \in \mathfrak{U}$ and $S \subseteq T$, then $T \in \mathfrak{U}$; and

(U4) either $S \in \mathfrak{U}$ or $\Lambda \backslash S \in \mathfrak{U}$ for all $S \subseteq \Lambda$.

Recall that a family $\mathfrak{F}$ of subsets of $\Lambda$ satisfying properties (U1) (U3) is called a filter on $\Lambda$, and a family $\mathfrak{B}$ of subsets of $\Lambda$ satisfying properties (U1) - (U2) is called a filter base. Each filter base generates a filter and each filter can be extended to an ultrafilter.

Remark 2.2 It follows from (U1) - (U4) that if $\mathfrak{U}$ is an ultrafilter on $\Lambda$ and $\left\{\Lambda_{1}, \ldots, \Lambda_{n}\right\}$ is a partition of $\Lambda$, then $\Lambda_{i} \in \mathfrak{U}$ for a unique $i \in$ $\{1, \ldots, n\}$. In particular, by (3) and (U4), if $\mathfrak{U}$ is an ultrafilter on $\Lambda$ and $g \in A(\Lambda)$, then exactly one of $S_{+}(g)$, fix $(g)$ and $S_{-}(g)$ belongs to $\mathfrak{U}$.

Let $\mathcal{U}=\left\{\mathfrak{U}_{\alpha} \mid \alpha \in A\right\}$ denote the set of all ultrafilters on $\Lambda$ and let $\preceq$ be a well ordering of $\mathcal{U}$. We can define a right order on an $\ell$-group $G \leq \mathrm{A}(\Lambda)$ (associated with $(\mathcal{U}, \preceq)$ ) as follows.

Let $G \leq \mathrm{A}(\Lambda)$ and $g \in G$. If $g \neq 1$, then $\operatorname{supp}(g) \neq \emptyset$. So there is an ultrafilter $\mathfrak{U}_{0}$ on $\Lambda$ containing $\operatorname{supp}(g)$; thus $\operatorname{fix}(g) \notin \mathfrak{U}_{0}$ and either $S_{+}(g) \in \mathfrak{U}_{0}$ or $S_{-}(g) \in \mathfrak{U}_{0}$ (and not both). Thus there is an ultrafilter $\mathfrak{U}_{0}$ such that $\operatorname{fix}(g) \in \mathfrak{U}$ for all $\mathfrak{U} \prec \mathfrak{U}_{0}$ and $\operatorname{supp}(g) \in \mathfrak{U}_{0}$. Define $g>1$ if $S_{+}(g) \in \mathfrak{U}_{0}$ and $g<1$ if $S_{-}(g) \in \mathfrak{U}_{0}$. So $g>1$ or $g<1$ (and not both) if $g \neq 1$. Let $1<g, h \in G$. So there are ultrafilters $\mathfrak{U}_{1}$ and $\mathfrak{U}_{2}$ on $\Lambda$ such that $S_{+}(g) \in \mathfrak{U}_{1}$ and $\operatorname{fix}(g) \in \mathfrak{U}$ for all $\mathfrak{U} \prec \mathfrak{U}_{1}$, and $S_{+}(h) \in \mathfrak{U}_{2}$ and fix $(h) \in \mathfrak{U}$ for all $\mathfrak{U} \prec \mathfrak{U}_{2}$. Since fix $(g h) \supseteq \operatorname{fix}(g) \cap \operatorname{fix}(h)$, fix $(g h) \in \mathfrak{U}$ for all $\mathfrak{U} \prec \min _{\preceq}\left\{\mathfrak{U}_{1}, \mathfrak{U}_{2}\right\}$. If $\mathfrak{U}_{1}=\mathfrak{U}_{2}$, then $S_{+}(g h) \supseteq S_{+}(g) \cap S_{+}(h) \in \mathfrak{U}_{1}$, so $g h>1$. On the other hand, if $\mathfrak{U}_{1} \prec \mathfrak{U}_{2}$ (without loss of generality), then $S_{+}(g), \operatorname{fix}(h) \in \mathfrak{U}_{1}$. Since $S_{+}(g h), S_{+}(h g) \supseteq S_{+}(g) \cap \operatorname{fix}(h) \in \mathfrak{U}_{1}$, we have $g h>1$ and $h g>1$. This therefore gives a right order $\leq$ on $G$ which we call the right order associated with $(\mathcal{U}, \preceq)$. 
Remark 2.3 If $\mathfrak{U} \in \mathcal{U}$ and $g \in G$, then $\mathfrak{U} g=\{S g \subseteq \Lambda \mid S \in \mathfrak{U}\} \in \mathcal{U}$. So we can define a conjugate $\preceq^{g}(g \in G)$ of $\preceq$ by

$$
\mathfrak{U}_{\alpha} \preceq^{g} \mathfrak{U}_{\beta} \quad \Longleftrightarrow \quad \mathfrak{U}_{\alpha} g^{-1} \preceq \mathfrak{U}_{\beta} g^{-1} .
$$

We also see that if $\leq$ is a right order associated with $(\mathcal{U}, \preceq)$, then its conjugate right order $\leq^{g}$ (given by $f \leq \leq^{g} h$ if and only if $g f \leq g h$ ) is the right order associated with $\left(\mathcal{U}, \preceq^{g}\right)$.

Let $G_{i}$ be a group with subgroup $H_{i}(i=1,2)$. Suppose that $H_{1}$ and $H_{2}$ are isomorphic, say $\varphi: H_{1} \cong H_{2}$. An amalgam of groups $G_{1}, G_{2}$ (with isomorphic subgroups $H_{1}$ and $H_{2}$ amalgamated by $\varphi$ ) is a group $L$ with embeddings $\varepsilon_{i}: G_{i} \rightarrow L(i=1,2)$ such that $h_{1}^{\varepsilon_{1}}=h_{1}^{\varphi \varepsilon_{2}}$ for all $h_{1} \in H_{1}$. Amalgams of two groups always exist in the category of groups. When groups $G_{1}, G_{2}$ carry an additional structure (e.g., they are right-ordered, $\ell$-groups or o-groups), we require that their amalgam $L$ has the same structure and that the embeddings are also order or lattice embeddings. For (right- or totally) orderable groups, we only require that an amalgam is (right or totally) orderable.

An amalgam $L$ of $\ell$-groups $G_{1}, G_{2}$ with embeddings $\varepsilon_{i}: G_{i} \rightarrow L$ $(i=1,2)$ is called an $\ell$-amalgam if each $\varepsilon_{i}$ is an $\ell$-embedding $(i=1,2)$ (so $G_{i}^{\varepsilon_{i}}$ are $\ell$-subgroups of the $\ell$-group $L(i=1,2)$ ).

We distinguish between two versions of amalgams for lattice-orderable groups $G_{1}$ and $G_{2}$. We call the lattice-orderable group $L$ a $g$-amalgam if $\varepsilon_{i}$ are group embeddings. And if there are lattice orders on $L, G_{1}, G_{2}$ such that $L$ becomes an $\ell$-amalgam of $\ell$-groups $L, G_{1}, G_{2}$, then we call $L$ an $\mathcal{L}$-amalgam. Clearly, any $\mathcal{L}$-amalgam is a $g$-amalgam.

\section{Proofs of Theorems}

Proof of Theorem $A$ : Let $G_{i} \leq \mathrm{A}\left(\Lambda_{i}\right)$ be an $\ell$-group and $\Lambda_{i}$ satisfy (I) and (II) of Corollary $2.1(i=1,2)$. Let $H_{1} \stackrel{\varphi}{\cong} H_{2}$ be $\ell$-isomorphic $\ell$-subgroups of $G_{1}$ and $G_{2}$, respectively.

For $i \in\{1,2\}$, let $\mathcal{U}_{i}$ be the set of all ultrafilters on $\Lambda_{i}$ and $\left\{\preceq_{\kappa} \mid \kappa \in\right.$ $\left.K_{i}\right\}$ be the set of all well orderings of $\mathcal{U}_{i}$. Let $\mathcal{R}_{i}$ be set of all right orders on $G_{i}$ associated with $\left\{\preceq_{\kappa} \mid \kappa \in K_{i}\right\}$. As observed in Remark 2.3, $\mathcal{R}_{i}$ is 
closed under conjugate orders and so is a normal family of right orders on $G_{i}(i=1,2)$.

By Theorem A of [1] (stated in Section 1 above), it remains to show that $\left(\mathcal{R}_{1}, \mathcal{R}_{2}\right)$ is compatible for $\varphi$; i.e., we must verify conditions (1) and (2). To achieve this, we first establish a lemma.

Lemma 3.1 Let $\mathfrak{U}_{\alpha} \in \mathcal{U}_{1}$ and

$$
\operatorname{Fix}(\alpha)=\left\{h \in H_{1} \mid \operatorname{fix}(h) \in \mathfrak{U}_{\alpha}\right\} .
$$

Then $\operatorname{Fix}(\alpha)$ is an $\ell$-subgroup of $G_{1}$ and $\operatorname{Conv}_{G_{1}}(\operatorname{Fix}(\alpha)) \cap H_{1}=\operatorname{Fix}(\alpha)$.

Proof: Clearly, $\operatorname{Fix}(\alpha)$ is a subgroup of $H_{1}$. Moreover, as $\operatorname{fix}(h \vee 1) \supseteq$ fix $(h)$, we have $h \vee 1 \in \operatorname{Fix}(\alpha)$ whenever $h \in \operatorname{Fix}(\alpha)$. Hence $\operatorname{Fix}(\alpha)$ is an $\ell$-subgroup of $H_{1}$. But $\operatorname{fix}(|h|) \subseteq \operatorname{fix}(|g|) \subseteq \operatorname{fix}(g)$ if $h \in H_{1}, g \in G_{1}$ and $|g| \leq|h|$. Thus $g \in \operatorname{Conv}_{G_{1}}(\operatorname{Fix}(\alpha))$ implies that $\operatorname{fix}(g) \in \mathfrak{U}_{\alpha}$. The lemma follows. /I

Since $\varphi$ is an $\ell$-isomorphism between $H_{1}$ and $H_{2}$, we immediately deduce

Corollary 3.2 If $\mathfrak{U}_{\alpha}$ is an ultrafilter on $\Lambda_{1}$, then $\operatorname{Conv}_{G_{2}}\left(\operatorname{Fix}(\alpha)^{\varphi}\right) \cap H_{2}=$ $\operatorname{Fix}(\alpha)^{\varphi}$.

Let $\leq_{1} \in \mathcal{R}_{1}$. By construction, $\leq_{1}$ is associated with a well ordering $\preceq_{1}$ of $\mathcal{U}_{1}$. We seek a right order $\leq_{2} \in \mathcal{R}_{2}$ compatible with $\varphi$. We need to use $\preceq_{1}$ to define a "compatible" well order $\preceq_{2}$ on $\mathcal{U}_{2}$.

First, we define a function $\psi: \mathfrak{U}_{\alpha} \mapsto \mathfrak{B}_{\alpha}$ which associates with each ultrafilter $\mathfrak{U}_{\alpha} \in \mathcal{U}_{1}$ a filter base $\mathfrak{B}_{\alpha}$ in $\Lambda_{2}$. For this, let $\mathfrak{U}_{\alpha}$ be an ultrafilter on $\Lambda_{1}$. Let

$S_{+}(\alpha):=\left\{h_{1} \in H_{1} \mid S_{+}\left(h_{1}\right) \in \mathfrak{U}_{\alpha}\right\}, \quad S_{-}(\alpha):=\left\{h_{1} \in H_{1} \mid S_{-}\left(h_{1}\right) \in \mathfrak{U}_{\alpha}\right\}$.

By Remark 2.2, $\left\{S_{+}(\alpha), \operatorname{Fix}(\alpha), S_{-}(\alpha)\right\}$ is a partition of $H_{1}$.

We now define a filter base $\mathfrak{B}_{\alpha}$ on $\Lambda_{2}$ associated with the ultrafilter $\mathfrak{U}_{\alpha}$ on $\Lambda_{1}$. Since $\varphi$ is an $\ell$-isomorphism, $\left\{S_{-}(\alpha)^{\varphi}, \operatorname{Fix}(\alpha)^{\varphi}, S_{+}(\alpha)^{\varphi}\right\}$ is a partition of $H_{2}$. Let $\mathfrak{B}_{\alpha}$ be the set of finite intersections of sets belonging to

$\left\{S_{+}\left(h_{2}\right) \mid h_{2} \in S_{+}(\alpha)^{\varphi}\right\} \cup\left\{\operatorname{fix}\left(h_{2}\right) \mid h_{2} \in \operatorname{Fix}(\alpha)^{\varphi}\right\} \cup\left\{S_{-}\left(h_{2}\right) \mid h_{2} \in S_{-}(\alpha)^{\varphi}\right\}$. 
We show that $\emptyset \notin \mathfrak{B}_{\alpha}$.

Now $h_{2} \in S_{k}(\alpha)^{\varphi}$ if and only if $S_{k}\left(h_{2}^{\varphi^{-1}}\right) \in \mathfrak{U}_{\alpha}(k \in\{ \pm\})$. Since $\emptyset \notin \mathfrak{U}_{\alpha}$ and $\varphi$ is an $\ell$-isomorphism, $S_{k}\left(h_{2}\right) \neq \emptyset$ if $S_{k}\left(h_{2}\right) \in \mathfrak{B}_{\alpha}(k \in\{ \pm\})$. Also, fix $\left(h_{2}\right) \neq \emptyset$ for all $h_{2} \in H_{2}$ by Corollary 2.1(I). So the empty set is not a member of the union used to form $\mathfrak{B}_{\alpha}$.

By Corollary 2.1(I), $\bigcap_{j=1}^{n} \operatorname{fix}\left(f_{j}\right) \neq \emptyset$ for all $f_{1}, \ldots, f_{n} \in H_{2}$.

Assume that $S_{+}\left(f_{1}\right), \ldots, S_{+}\left(f_{n}\right) \in \mathfrak{B}_{\alpha}$. Then $f_{1}, \ldots, f_{n} \in S_{+}(\alpha)^{\varphi}$ and so $f_{1}^{\varphi^{-1}}, \ldots, f_{n}^{\varphi^{-1}} \in S_{+}(\alpha)$. Hence $S_{+}\left(f_{1}^{\varphi^{-1}}\right), \ldots, S_{+}\left(f_{n}^{\varphi^{-1}}\right) \in \mathfrak{U}_{\alpha}$, whence $\bigcap_{j=1}^{n} S_{+}\left(f_{j}^{\varphi^{-1}}\right) \neq \emptyset$. Thus $\left(f_{1}^{\varphi^{-1}}\right)^{+} \wedge \ldots \wedge\left(f_{n}^{\varphi^{-1}}\right)^{+} \neq 1$. Therefore, $f_{1}^{+} \wedge \ldots \wedge f_{n}^{+} \neq 1$ in $G_{2}$. Consequently, $\bigcap_{j=1}^{n} S_{+}\left(f_{j}\right) \neq \emptyset$.

By replacing $f_{1}, \ldots, f_{n}$ by their inverses, the same argument applies if $S_{-}\left(f_{1}\right), \ldots, S_{-}\left(f_{n}\right) \in \mathfrak{B}_{\alpha}$. The analogous argument applies when $S_{+}\left(f_{1}\right), \ldots, S_{+}\left(f_{m}\right), S_{-}\left(f_{m+1}\right), \ldots, S_{-}\left(f_{n}\right) \in \mathfrak{B}_{\alpha}$.

By the above technique, to complete the proof that $\mathfrak{B}_{\alpha}$ is a filter base, it suffices to show that fix $\left(h_{0}\right) \cap S_{+}\left(h_{2}\right) \in \mathfrak{B}_{\alpha}$ whenever fix $\left(h_{0}\right), S_{+}\left(h_{2}\right) \in$ $\mathfrak{B}_{\alpha}$. Let $h_{0}, h_{2} \in H_{2}$ be such that fix $\left(h_{0}\right), S_{+}\left(h_{2}\right) \in \mathfrak{B}_{\alpha}$. By the construction, $h_{0}^{\varphi^{-1}} \in \operatorname{Fix}(\alpha), h_{2}^{\varphi^{-1}} \in S_{+}(\alpha)$; so fix $\left(h_{0}^{\varphi^{-1}}\right), S_{+}\left(h_{2}^{\varphi^{-1}}\right) \in \mathfrak{U}_{\alpha}$. By Lemma 3.1, $\operatorname{Fix}(\alpha)$ is the $\ell$-subgroup of $G_{1}$ and $\operatorname{Conv}_{G_{1}}(\operatorname{Fix}(\alpha)) \cap H_{1}=$ $\operatorname{Fix}(\alpha)$. By Corollary 3.2, the analogous result is true for $\operatorname{Fix}(\alpha)^{\varphi}$ in $G_{2}$. But $\left(h_{2}^{\varphi^{-1}}\right)^{+} \notin \operatorname{Fix}(\alpha)$ and so, by Lemma 3.1, $\left(h_{2}^{\varphi^{-1}}\right)^{+} \not \leq|h|$ for any $h \in \operatorname{Fix}(\alpha)$, i.e., $\left(h_{2}^{\varphi^{-1}}\right)^{+} \vee|h| \neq|h|$ for all $h \in \operatorname{Fix}(\alpha)$. Applying the $\ell$-isomorphism $\varphi$, we obtain $h_{2}^{+} \vee|h| \neq|h|$ for all $h \in \operatorname{Fix}(\alpha)^{\varphi}$. Thus $h_{2}^{+} \notin \operatorname{Conv}_{G_{2}}\left(\operatorname{Fix}(\alpha)^{\varphi}\right)$ and by Corollary 2.1(I), $S_{+}\left(h_{2}\right) \cap \operatorname{fix}\left(h_{0}\right) \neq \emptyset$, as desired. This completes the proof that $\mathfrak{B}_{\alpha}$ is a filter base on $\Lambda_{2}$.

Therefore, the map $\psi: \mathfrak{U}_{\alpha} \mapsto \mathfrak{B}_{\alpha}(\alpha \in A)$ is defined. However, in general, $\psi$ need not be a one-to-one correspondence. We therefore take the subset $A_{1}$,

$$
A_{1}:=\left\{\alpha \in A \mid \alpha=\min _{\preceq}\left\{\beta \in A \mid \mathfrak{U}_{\beta}^{\psi}=\mathfrak{U}_{\alpha}^{\psi}\right\}\right\}
$$

of $A$ so that the restriction of $\psi$ to $\left\{\mathfrak{U}_{\alpha} \mid \alpha \in A_{1}\right\}$ is a one-to-one correspondence and $\left\{\mathfrak{U}_{\alpha}^{\psi} \mid \alpha \in A_{1}\right\}=\left\{\mathfrak{U}_{\alpha}^{\psi} \mid \alpha \in A\right\}$.

For each $\alpha \in A_{1}$, we extend $\mathfrak{B}_{\alpha}$ to an ultrafilter $\mathfrak{V}_{\alpha}$ on $\Lambda_{2}$ and transfer the well-ordering $\preceq_{1}$ from $\left\{\mathfrak{U}_{\alpha} \mid \alpha \in A_{1}\right\}$ to $\mathcal{V}_{1}=\left\{\mathfrak{V}_{\alpha} \mid \alpha \in A_{1}\right\}$ :

$$
\mathfrak{V}_{\alpha} \preceq_{2} \mathfrak{V}_{\beta} \quad \Longleftrightarrow \quad \mathfrak{U}_{\alpha} \preceq_{1} \mathfrak{U}_{\beta} .
$$


We enumerate $\mathcal{U}_{2}$, the set of all ultrafilters on $\Lambda_{2}$, say $\mathcal{U}_{2}:=\left\{\mathfrak{V}_{\beta} \mid \beta \in B\right\}$, where $B \supseteq A_{1}$. If $\mathcal{U}_{2} \neq \mathcal{V}_{1}$, let $\preceq_{2}$ be an arbitrary well-ordering of $\mathcal{V}_{2}:=\mathcal{U}_{2} \backslash \mathcal{V}_{1}$. Finally, extend the well-order $\preceq_{2}$ to all of $\mathcal{U}_{2}$ by putting

$$
\mathfrak{V}_{\alpha} \prec_{2} \mathfrak{V}_{\beta} \quad \text { for all } \mathfrak{V}_{\alpha} \in \mathcal{V}_{1}, \mathfrak{V}_{\beta} \in \mathcal{V}_{2}
$$

The right orders $\leq_{1}$ on $G_{1}$ associated with $\left(\mathcal{U}_{1}, \preceq_{1}\right)$ and $\leq_{2}$ on $G_{2}$ associated with $\left(\mathcal{U}_{2}, \preceq_{2}\right)$ satisfy the compatibility conditions (1) as we now show. Let $h_{1} \in H_{1}$. If $1<_{1} h_{1}$, then by the definition of $\leq_{1}$ there is an ultrafilter $\mathfrak{U}_{\alpha} \in \mathcal{U}_{1}$ such that $S_{+}\left(h_{1}\right) \in \mathfrak{U}_{\alpha}$ and $\operatorname{fix}\left(h_{1}\right) \in \mathfrak{U}_{\beta}$ for all $\mathfrak{U}_{\beta} \prec_{1} \mathfrak{U}_{\alpha}$. By construction, $S_{+}\left(h_{1}^{\varphi}\right) \in \mathfrak{B}_{\alpha}$ and $\operatorname{fix}\left(h_{1}^{\varphi}\right) \in \mathfrak{B}_{\beta}$ for all $\mathfrak{B}_{\beta}=\mathfrak{U}_{\beta}^{\psi}$ when $\mathfrak{U}_{\beta} \prec_{1} \mathfrak{U}_{\alpha}$. It follows that $\alpha=\min _{\preceq}\left\{\gamma \in A \mid \mathfrak{U}_{\gamma}^{\psi}=\mathfrak{U}_{\alpha}^{\psi}\right\}$. Hence $\alpha \in A_{1}$ and $\mathfrak{B}_{\alpha} \subseteq \mathfrak{V}_{\alpha} \in \mathcal{V}_{1}$. Thus $S_{+}\left(h_{1}^{\varphi}\right) \in \mathfrak{V}_{\alpha} \in \mathcal{V}_{1}$ and $\operatorname{fix}\left(h_{1}^{\varphi}\right) \in \mathfrak{V}_{\beta}$ for all $\mathfrak{V}_{\beta} \prec_{2} \mathfrak{V}_{\alpha}$. By the definition of the right order $<_{2}$ associated with $\left(\mathcal{U}_{2}, \preceq_{2}\right)$, we get $h_{1}^{\varphi}>_{2} 1$. By symmetry, condition (2) also holds and the proof of the theorem is complete. //

Proof of Theorem $B$ : If $G_{1} * G_{2}\left(H_{1} \stackrel{\varphi}{\cong} H_{2}\right)$ is right orderable, then there is a group embedding of $G_{1} * G_{2}\left(H_{1} \stackrel{\varphi}{\cong} H_{2}\right)$ into an $\ell$-group $L$. So there are group embeddings $\varepsilon_{i}: G_{i} \rightarrow L(i=1,2)$ such that $h_{1}^{\varphi \varepsilon_{2}}=h_{1}^{\varepsilon_{1}}$ for all $h_{1} \in H_{1}$. Since $\ell$-subgroups $\left\langle H_{1}^{\varepsilon_{1}}\right\rangle_{\ell}$ and $\left\langle H_{2}^{\varepsilon_{2}}\right\rangle_{\ell}$ of $L$ generated by $H_{1}^{\varepsilon_{1}}$ and $H_{2}^{\varepsilon_{2}}$ coincide, we can take $\hat{\varphi}$ to be the identity mapping on $L$.

Conversely, suppose that there are embeddings $\varepsilon_{i}$ of $G_{i}$ in $\ell$-groups $\hat{G}_{i}(i=1,2)$ and an $\ell$-isomorphism $\hat{\varphi}$ between $\hat{H}_{1}:=\left\langle H_{1}^{\varepsilon_{1}}\right\rangle_{\ell}$ and $\hat{H}_{2}:=$ $\left\langle H_{2}^{\varepsilon_{2}}\right\rangle_{\ell}$ such that $h_{1}^{\varphi \varepsilon_{2}}=h_{1}^{\varepsilon_{1} \hat{\varphi}}$ for all $h_{1} \in H_{1}$. Then the group $\hat{G}_{1} *$ $\hat{G}_{2}\left(\hat{H}_{1} \stackrel{\hat{\vartheta}}{\cong} \hat{H}_{2}\right)$ is right orderable by Theorem A and contains an isomorphic copy of $G_{1} * G_{2}\left(H_{1} \stackrel{\varphi}{\cong} H_{2}\right)$ by construction. //

\section{Amalgamations and prime $\ell$-ideals}

Proposition 4.1 Let $G_{i}$ be a lattice-ordered group and $H_{i}$ be a prime $\ell$-ideal of $G_{i}(i=1,2)$. Suppose that $\varphi: H_{1} \cong H_{2}$ is an $\ell$-isomorphism. Then $L=G_{1} * G_{2}\left(H_{1} \stackrel{\varphi}{\cong} H_{2}\right)$ is lattice-orderable $\mathcal{L}$-amalgam of groups $G_{1}, G_{2}$ with amalgamated subgroups $H_{1}, H_{2}$. 
Proof: Let $i \in\{1,2\}$. Denote by $H_{i}^{+}$the set of positive elements of $H_{i}$ under the original lattice order on $G_{i}$ and denote by $\bar{G}_{i}^{+}$the set of positive elements of $G_{i} / H_{i}$ under the order induced on $G_{i} / H_{i}$ by the original lattice orders on $G_{i}$. This induces a lexicographic lattice order on $G_{i}$ whose set of positive elements is

$$
P_{i}=\left\{g \in G_{i} \mid H_{i} g_{i} \in \bar{G}_{i}^{+} \backslash\left\{H_{i}\right\} \text { or } g \in H_{i}^{+}\right\}
$$

By (4), $H_{i}$ is also a prime $\ell$-ideal of $G_{i}$ under this new lattice ordering. Moreover, $P_{i} \cap H_{i}=H_{i}^{+}$and the new order on $H_{i}$ coincides with the old one. Thus $\varphi$ is also an $\ell$-isomorphism with respect to the new orders on $H_{1}$ and $H_{2}$. We can extend the total orders from $G_{1} / H_{1}$ and $G_{2} / H_{2}$ to the free product $G_{1} / H_{1} * G_{2} / H_{2}$ (see [7]). Since $L / H_{i}^{\varepsilon_{i}} \cong G_{1} / H_{1} * G_{2} / H_{2}$ and the order on $H_{i}^{\varepsilon_{i}}$ is invariant under conjugation by elements from $L$ (since $L$ is generated by $G_{1}^{\varepsilon_{1}} \cup G_{2}^{\varepsilon_{2}}$ ), we can obtain a lexicographic lattice order on $L$. Let $P$ be the set of positive elements of $L$ under this ordering. Now $P \cap G_{i}=P_{i}(i=1,2)$. Let $i \in\{1,2\}$ and $g \in G_{i}^{\varepsilon_{i}}$. If $g \in H_{i}^{\varepsilon_{i}}$, then $g \vee 1 \in H_{i}^{\varepsilon_{i}}$ since $H_{i}^{\varepsilon_{i}}$ is an $\ell$-ideal of $L$. If $g \in G_{i}^{\varepsilon_{i}} \backslash H_{i}^{\varepsilon_{i}}$, either $g$ is strictly positive or strictly negative in $L$; thus $g \vee 1 \in G_{i}^{\varepsilon_{i}}$. Consequently, $G_{i}^{\varepsilon_{i}}$ is an $\ell$-subgroup of $\ell$-group $L$ and the proposition is proved. //

A proof of the existence of an $\ell$-amalgam is possible in the special case when the $\ell$-groups are splitting extensions of the prime $\ell$-ideals; considering it leads to Example 5.1.

Let $G_{i}=H_{i} \overleftarrow{\rtimes} K_{i}$ for $K_{i}$ an o-group and $H_{i}$ an $\ell$-group $(i=1,2)$ - so $G_{i}$ is an $\ell$-group $(i=1,2)$. Suppose that $\varphi: H_{1} \cong H_{2}$ is an $\ell$ isomorphism. We say that the extensions $K_{1}$ and $K_{2}$ are compatible for $\varphi$ if for all $k_{i} \in K_{i}(i=1,2)$ and all $h_{1} \in H_{1}$,

$$
\left[\left(\left(h_{1}^{k_{1}}\right)^{\varphi}\right)^{k_{2}}\right]^{\varphi^{-1}}=\left[\left(\left(h_{1}^{\varphi}\right)^{k_{2}}\right)^{\varphi^{-1}}\right]^{k_{1}} .
$$

Proposition 4.2 Let $G_{i}=H_{i} \overleftarrow{\rtimes} K_{i}$ for $K_{i}$ an o-group and $H_{i}$ a latticeordered group $(i=1,2)$. Suppose that $\varphi: H_{1} \cong H_{2}$ is an $\ell$-isomorphism and $K_{1}$ and $K_{2}$ are compatible for $\varphi$. Then there is a lattice-ordered group $L$ and $\ell$-embeddings $\varepsilon_{i}: G_{i} \rightarrow L(i=1,2)$ such that $g_{1}^{\varepsilon_{1}}=g_{2}^{\varepsilon_{2}}$ if and only if $g_{1} \in H_{1}$ and $g_{2}=g_{1}^{\varphi}\left(g_{i} \in G_{i}, i=1,2\right)$.

Proof: Let $L:=\left(H_{2} \overleftarrow{\rtimes} K_{2}\right) \overleftarrow{\rtimes} K_{1}$, where the action of $k_{1} \in K_{1}$ on $\left(h_{2}, k_{2}\right)$ is given by

$$
\left(h_{2}, k_{2}\right)^{k_{1}}:=\left(\left(\left(h_{2}^{\varphi^{-1}}\right)^{k_{1}}\right)^{\varphi}, k_{2}\right) \quad\left(h_{2} \in H_{2}, k_{2} \in K_{2}\right) .
$$


This is well-defined as the extensions are compatible for $\varphi$. Now $L$ is an $\ell$-group and $\varepsilon_{2}: G_{2} \rightarrow L$ given by $\left(h_{2}, k_{2}\right) \mapsto\left(h_{2}, k_{2}, 1\right)$ is an $\ell$ embedding. Let $\varepsilon_{1}: G_{1} \rightarrow L$ be given by $\left(h_{1}, k_{1}\right) \mapsto\left(h_{1}^{\varphi}, 1, k_{1}\right)$. Then $\varepsilon_{1}$ is a well-defined $\ell$-embedding by compatibility and the definition of the splitting extension of $G_{2}=H_{2} \overleftarrow{\rtimes} K_{2}$ by $K_{1}$. Moreover, if $g_{i}=\left(h_{i}, k_{i}\right)$ $(i=1,2)$, then $g_{1}^{\varepsilon_{1}}=g_{2}^{\varepsilon_{2}}$ if and only if $\left(h_{1}^{\varphi}, 1, k_{1}\right)=\left(h_{2}, k_{2}, 1\right)$ if and only if $\left(k_{1}=1=k_{2}\right.$ and $\left.h_{2}=h_{1}^{\varphi}\right)$. //

Note that if, say, $K_{1}$ is a direct extension of $H_{1}$, then the compatibility condition automatically holds for any split extension of $H_{2}$ by $K_{2}$ and so the lemma applies. However, we can do better in this case; $G_{2}$ can be an arbitrary $\ell$-group with $H_{2}$ not necessarily convex or normal or prime in $G_{2}$.

Proposition 4.3 Let $G_{i}$ be a lattice-ordered group with $\ell$-subgroup $H_{i}$ $(i=1,2)$. If $\varphi: H_{1} \cong H_{2}$ is an l-isomorphism and $G_{1}=H_{1} \overleftarrow{\times} K_{1}$ for some o-group $K_{1}$, then there is an $\ell$-group $L$ and $\ell$-embeddings $\varepsilon_{i}$ : $G_{i} \rightarrow L(i=1,2)$ such that $g_{1}^{\varepsilon_{1}}=g_{2}^{\varepsilon_{2}}$ if and only if $g_{1} \in H_{1}$ and $g_{2}=g_{1}^{\varphi}$ $\left(g_{i} \in G_{i}, i=1,2\right)$.

Proof: Let $L:=G_{2} \overleftarrow{\times} K_{1}$, an $\ell$-group, and $\varepsilon_{2}: G_{2} \rightarrow L$ be given by $g_{2} \mapsto\left(g_{2}, 1\right) \in L$. Clearly, $\varepsilon_{2}$ is an $\ell$-embedding. Let $\varepsilon_{1}: G_{1} \rightarrow L$ be given by $\left(h_{1}, k_{1}\right) \mapsto\left(h_{1}^{\varphi}, k_{1}\right)$. Then $\varepsilon_{1}$ is also an $\ell$-embedding. Now $g_{1}^{\varepsilon_{1}}=g_{2}^{\varepsilon_{2}}$ if and only if $g_{1}=\left(h_{1}, 1\right)$ for some $h_{1} \in H_{1}$ and $g_{2}=h_{1}^{\varphi}$; i.e., $g_{1}^{\varphi}$ is defined and equal to $g_{2}$. //

Remark 4.4 Pierce proved in [5] that $\ell$-amalgams always exist when the amalgamated $\ell$-subgroups are cyclic and generated by a positive element. If we take an element $h_{i}$ unrelated to the identity in $G_{i}$, the $\ell$-subgroup generated by $h_{i}$ is generated by the disjoint elements $h_{i}^{+}$and $h_{i}^{-}(i=1,2)$. Pierce's proof ( $i b i d)$ can be adapted to give an $\ell$-group $L$ containing $G_{1}$ and $G_{2}$ as $\ell$-subgroups such that one can simultaneously conjugate $h_{1}^{+}$ to $h_{2}^{+}$and $h_{1}^{-}$to $h_{2}^{-}$in $L$; so the $\ell$-subgroups generated by $h_{1}$ and $h_{2}$ can be $\ell$-amalgamated in this case, too. In contrast, Pierce's example (ibid) or Example 5.1 below shows that an $\ell$-amalgam need not exist if the amalgamated $\ell$-subroups are abelian and generated by two elements. 


\section{$5 \quad$ Limiting examples}

In [5], K. R. Pierce gave an example to show that, in general, $\ell$-amalgams do not exist. In this section, we show that such failure can occur even when the amalgamated $\ell$-subgroups are $\ell$-ideals. In the second example, we show that this failure can occur for all lattice orders on the constituent

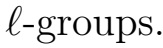

Example 5.1 There are lattice-ordered groups $G_{i}$ with prime $\ell$-ideals $H_{i}$ $(i=1,2)$ such that $\varphi: H_{1} \cong H_{2}$ is an $\ell$-isomorphism, but there is no $\ell$-amalgam for these $\ell$-groups.

The idea is to use splitting extensions that are incompatible for $\varphi$.

Let $A_{23}=\left\langle a_{2}\right\rangle \times\left\langle a_{3}\right\rangle$ be the cardinal direct product of two cyclic groups $\left\langle a_{2}\right\rangle$ and $\left\langle a_{3}\right\rangle$ with $a_{2}, a_{3}>1$, and $B_{1}=A_{23} \overleftarrow{\rtimes}\left\langle b_{1}\right\rangle$ be the lexicographic split extension of $A_{23}$ by the cyclic group $\left\langle b_{1}\right\rangle$ given by the automorphism $a_{2}^{b_{1}}=a_{3}, a_{3}^{b_{1}}=a_{2}$. Then $B_{1}$ is an $\ell$-group with $b_{1}>a$ for all $a \in A_{23}$. Let $G_{1}=B_{1} \times\left\langle a_{1}\right\rangle$ be the cardinal direct product of $B_{1}$ and the cyclic group $\left\langle a_{1}\right\rangle$ with $\left.a_{1}\right\rangle$. Similary, let $A_{13}=\left\langle a_{1}\right\rangle \times\left\langle a_{3}\right\rangle$ be the cardinal product and $B_{2}=A_{13} \overleftarrow{\rtimes}\left\langle b_{2}\right\rangle$ be the splitting extension of $A_{13}$ given by the automorphism $a_{1}^{b_{2}}=a_{3}, a_{3}^{b_{2}}=a_{1}$ with $b_{2}>a$ for all $a \in A_{13}$. Let $G_{2}=B_{2} \times\left\langle a_{2}\right\rangle$ be the cardinal product. Then $H_{1}=A_{23} \times\left\langle a_{1}\right\rangle$ and $H_{2}=A_{13} \times\left\langle a_{2}\right\rangle$ are prime $\ell$-ideals of the $\ell$-groups $G_{1}$ and $G_{2}$ respectively. The identity map $\varphi: a_{i} \mapsto a_{i}(i=1,2,3)$ is an $\ell$-isomorphism between $H_{1}$ and $H_{2}$. We claim that there is no $\ell$-group $L$ admitting $\ell$-embeddings $\varepsilon_{i}: G_{i} \rightarrow L(i=1,2)$ such that $h_{1}^{\varepsilon_{1}}=h_{1}^{\varphi \varepsilon_{2}}$ for all $h_{1} \in H_{1}$.

First note that $a_{1}, a_{2}, a_{3}$ are pairwise orthogonal and that $a_{i}$ is orthogonal to $b_{i}(i=1,2)$ :

$$
a_{1} \wedge a_{2}=a_{1} \wedge a_{3}=a_{2} \wedge a_{3}=1, \quad a_{1} \wedge b_{1}=a_{2} \wedge b_{2}=1 .
$$

For reductio ad absurdum, suppose that an $\ell$-amalgam $L$ were to exist. By the Holland Embedding Theorem (Theorem 2), represent $L$ by orderpreserving permutations of a totally ordered set $(\Lambda, \leq)$. Since $a_{2}^{b_{1}^{ \pm 1}}=a_{3}$ and orthogonal elements have disjoint supports, we have

$$
\operatorname{supp}\left(a_{2}\right) \cup \operatorname{supp}\left(a_{3}\right) \subseteq \operatorname{supp}\left(b_{1}\right) \quad \text { and } \operatorname{supp}\left(a_{1}\right) \subseteq \operatorname{fix}\left(b_{1}\right) .
$$

Similary,

$$
\operatorname{supp}\left(a_{1}\right) \cup \operatorname{supp}\left(a_{3}\right) \subseteq \operatorname{supp}\left(b_{2}\right) \quad \text { and } \quad \operatorname{supp}\left(a_{2}\right) \subseteq \operatorname{fix}\left(b_{2}\right) .
$$


It follows that

$$
\operatorname{supp}\left(a_{1}\right) b_{2}^{ \pm 1}=\operatorname{supp}\left(a_{3}\right) \quad \text { and } \quad \operatorname{supp}\left(a_{2}\right) b_{1}^{ \pm 1}=\operatorname{supp}\left(a_{3}\right) .
$$

Take $\lambda_{1} \in \operatorname{supp}\left(a_{1}\right)$ and let $\lambda_{3}:=\lambda_{1} b_{2}, \lambda_{2}:=\lambda_{3} b_{1}^{-1}$. Then $\lambda_{1}, \lambda_{2}<\lambda_{3}$ (since $b_{1}, b_{2}>1$ ) and $\lambda_{1}<\lambda_{2}$ (since $\lambda_{1} \in \operatorname{fix}\left(b_{1}\right)$ ). Hence $\lambda_{1}<\lambda_{2}<\lambda_{3}$. By (5) $\lambda_{2} \in \operatorname{supp}\left(a_{2}\right) \subseteq \operatorname{fix}\left(b_{2}\right)$; so $\lambda_{3}=\lambda_{1} b_{2}<\lambda_{2}$ - the desired contradiction. //

By Proposition 4.1, there is an $\mathcal{L}$-amalgam for the $\ell$-groups considered in Example 5.1. If we want to build an example of lattice-orderable groups which have no $\mathcal{L}$-amalgams, we must weaken the hypotheses of Proposition 4.1. As we now show, this is possible even when both amalgamated subgroups are $\ell$-ideals and one of them is prime (see Example 5.2 below).

Example 5.2 There are lattice-ordered groups $G_{i}$ with $\ell$-ideals $H_{i}(i=$ $1,2)$ such that $\varphi: H_{1} \cong H_{2}$ is an $\ell$-isomorphism, but there is no $\mathcal{L}$ amalgam for these lattice-orderable groups.

Corollary 1.1 guarantees the existence of lattice-orderable $g$-amalgams. But for any lattice order on such an amalgam $L$ in this example, at least one of images of $G_{1}$ and $G_{2}$ cannot be a sublattice of the $\ell$-group $L$.

To prove the result, we recall a lemma.

Lemma 5.3 (Holland and Scrimger [4]). If $g_{1} \wedge g_{2}=1, h_{1} \wedge h_{2}=1$ for elements $g_{1}, g_{2}, h_{1}, h_{2}$ in an $\ell$-group $G$, then $\left[h_{1}, g_{2}^{-1} h_{2} g_{1} h_{2}^{-1} g_{2} h_{2} g_{1}^{-1}\right]=1$.

We now construct the required $\ell$-groups and their $\ell$-ideals.

Let $A$ be the cardinal direct product of sixty eight cyclic $\ell$-groups

$$
A_{i, j}:=\left\langle a_{i, j} \mid a_{i, j} \vee 1=a_{i, j}\right\rangle, \quad 1 \leq i \leq 4,0 \leq j \leq 16 .
$$

So all $\ell$-groups $A_{i, j}$ are cyclic totally ordered groups with positive generators $a_{i, j}$ and $a_{1,0}^{n_{1,0}} \ldots a_{4,15}^{n_{4,15}}>1$ in $A$ if and only if all the exponents $n_{i, j}$ are non-negative and at least one $n_{i, j}$ is not equal to 0 .

Let $A_{1}$ be the $\ell$-subgroup of $A$ generated by the elements

$$
a_{1, j} \quad(j=0,1,8,9,16), \quad a_{2, j} \quad(j=2,3,4,5,6,7,10,11,12,13,14,15)
$$




$$
a_{3, j} \quad(j=0,1,2,5,6,11,12,15,16), \quad a_{4, j} \quad(j=3,4,7,8,9,10,13,14) .
$$

Let $A_{2}$ be the $\ell$-subgroup generated by the remaining generators. Thus $A=A_{1} \times A_{2}$, the cardinal product of $A_{1}$ and $A_{2}$.

We define $\ell$-automorphisms $\beta_{k}(0 \leq k \leq 15), \gamma, \delta$ on $\mathrm{A}$ and $b_{m}$ on $A_{m}(m=1,2)$. It suffices to define the requisite $\ell$-automorphisms on the generators $a_{i, j}$. Let

$$
\begin{gathered}
\beta_{k}: a_{i, k} \mapsto a_{i, k+1} \mapsto a_{i, k}, \quad a_{i, j} \mapsto a_{i, j} \quad(j \neq k, k+1) ; \\
\gamma: a_{1, j} \mapsto a_{2, j} \mapsto a_{1, j}, \quad a_{3, j} \mapsto a_{4, j} \mapsto a_{3, j} ; \\
\delta: a_{1, j} \mapsto a_{3, j} \mapsto a_{1, j}, \quad a_{2, j} \mapsto a_{4, j} \mapsto a_{2, j} ; \\
a_{1, j} \mapsto a_{1, j}^{\beta_{0} \beta_{8}}, \quad a_{2, j} \mapsto a_{2, j}^{\beta_{2} \beta_{4} \beta_{6} \beta_{10} \beta_{12} \beta_{14}}, \\
a_{3, j} \mapsto a_{3, j}^{\beta_{1} \beta_{5} \beta_{11} \beta_{15}}, \quad a_{4, j} \mapsto a_{4, j}^{\beta_{3} \beta_{7} \beta_{9} \beta_{13}} ; \\
b_{2}: a_{i, j} \mapsto a_{i, j}^{\gamma b_{1} \gamma} .
\end{gathered}
$$

By the definitions,

$$
A_{2}=A_{1}^{\gamma}, \quad A_{1}^{b_{1}}=A_{1}, \quad A_{2}^{b_{2}}=A_{2} .
$$

We build the lexicographic split extensions

$$
H_{1}:=A_{1} \overleftarrow{\rtimes}\left\langle b_{1}\right\rangle \quad \text { and } \quad H_{2}:=A_{2} \overleftarrow{\rtimes}\left\langle b_{2}\right\rangle
$$

prescribed by the automorphisms (6) and (7), respectively. Let

$$
H:=H_{1} \times H_{2},
$$

the cardinal product of $H_{1}$ and $H_{2}$, and build one more lexicographic split extension

$$
G_{1}:=H \overleftarrow{\rtimes}\langle c\rangle
$$

given by the automorphism

$$
c: a_{i, j} \mapsto a_{i, j}^{\gamma}, \quad b_{1} \mapsto b_{2} \mapsto b_{1}
$$


By $(6)-(8)$

$$
a_{i, j}^{b_{1}^{2}}=a_{i, j}^{b_{2}^{2}}=a_{i, j}^{c^{2}}=a_{i, j} \quad(1 \leq i \leq 4,0 \leq j \leq 16) .
$$

By construction, $G_{1}$ is an $\ell$-group with $b_{1} \wedge b_{2}=1$. Further, $A$ is an $\ell$-ideal in $G_{1}$ and each element of $G_{1}$ can be written uniquely in the form

$$
g=u b_{1}^{n} b_{2}^{m} c^{k} \quad \text { for some } u \in A, n, m, k \in \mathbb{Z} .
$$

Lemma 5.4 For any lattice ordering $\vee, \wedge$ on $G_{1}$, there are orthogonal elements $h_{1}, h_{2} \in G_{1}$ such that

$$
w^{h_{1}}=w^{b_{1}} \quad \text { and } \quad w^{h_{2}}=w^{b_{2}} \quad \text { for all } w \in A .
$$

Proof: Consider $b=b_{1} b_{2}^{-1}$. We have

$$
\left(b^{+}\right)^{c}=(b \vee 1)^{c}=b^{-1} \vee 1=b^{-} .
$$

So $b=b^{+}\left(b^{+}\right)^{-c}$. Write $b^{+}$in the normal form described in (10):

$$
b^{+}=u b_{1}^{n} b_{2}^{m} c^{k} .
$$

Since $b_{1} b^{+}=b^{+} b_{1}$, we must have that $k$ is even; say

$$
b^{+}=u b_{1}^{n} b_{2}^{m} c^{2 s} .
$$

Further,

$$
\left(b^{+}\right)^{c}=u^{c} b_{1}^{m} b_{2}^{n} c^{2 s}
$$

and

$$
\left(b^{+}\right)^{c}=b^{-}=b^{-1} b^{+}=u_{1} b_{1}^{n-1} b_{2}^{m+1} c^{2 s} .
$$

So $m=n-1$ and

$$
b^{+}=u b_{1}^{n} b_{2}^{n-1} c^{2 s}
$$

with $u \in A$.

If $n$ is odd, say $n=2 r+1$, let

$$
h_{1}=b^{+}=u b_{1}^{2 r} b_{2}^{2 r} c^{2 s} b_{1}, \quad h_{2}=b^{-}=u^{c} b_{1}^{2 r} b_{2}^{2 r} c^{2 s} b_{2} .
$$

If $n$ is even, say $n=2 r$, let

$$
h_{2}=b^{+}=u b_{1}^{2 r} b_{2}^{2 r-2} c^{2 s} b_{2}, \quad h_{1}=b^{-}=u^{c} b_{1}^{2 r-2} b_{2}^{2 r} c^{2 s} b_{1} .
$$


Thus $h_{1} \wedge h_{2}=b^{+} \wedge b^{-}=1$. By (9),

$$
w^{h_{1}}=w^{b_{1}} \quad w^{h_{2}}=w^{b_{2}}
$$

for all $w \in A$. /

We next construct the group $G_{2}$ as a lexicographic split extension of $A$ by an infinite cyclic group $\langle d\rangle$;

$$
G_{2}:=A \overleftarrow{\rtimes}\langle d\rangle
$$

where the action of $d$ on $A$ is defined by

$$
d: a_{i, j} \mapsto a_{i, j}^{\delta} .
$$

By construction, $A$ is a prime $\ell$-ideal of $G_{2}$.

Suppose that there are lattice orders on the groups $G_{1}, G_{2}$ such that there is an $\ell$-group $L$ with $\ell$-embeddings $\varepsilon_{1}: G_{1} \rightarrow L, \varepsilon_{2}: G_{1} \rightarrow L$ coinciding on $A$ (i.e. $a^{\varepsilon_{1}}=a^{\varepsilon_{2}}$ in $L$ for all $a \in A$ ). By Lemma 5.4, there are orthogonal elements $h_{1}, h_{2} \in G_{1}^{\varepsilon_{1}}<L$ such that

$$
a_{i, j}^{\varepsilon_{1} h_{1}}=a_{i, j}^{b_{1} \varepsilon_{1}}, \quad a_{i, j}^{\varepsilon_{1} h_{2}}=a_{i, j}^{b_{2} \varepsilon_{1}}
$$

for all $1 \leq i \leq 4,0 \leq j \leq 16$. Let $g_{1}=h_{1}^{d^{\varepsilon_{2}}}$ and $g_{2}=h_{2}^{d^{\varepsilon_{2}}}$. Then $g_{1}, g_{2}$ are also orthogonal. By Lemma 5.3,

$$
f=\left[h_{1}, g_{2}^{-1} h_{2} g_{1} h_{2}^{-1} g_{2} h_{2} g_{1}^{-1}\right]=1 .
$$

We calculate $a_{1,0}^{\varepsilon_{1} f}$ using (6), (7), and (11).

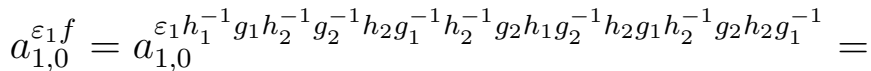

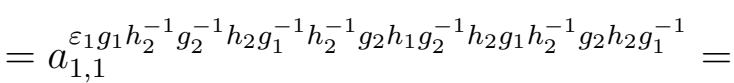

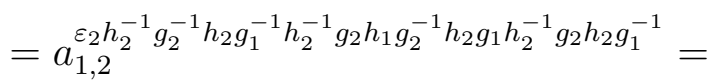

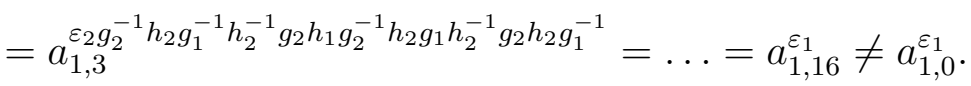

Consequently, $f \neq 1$ which contradicts (12). This establishes the claim of this example. // 


\section{$6 \quad$ Intersections of right orders}

By an analysis of $\ell$-groups which have an $\mathcal{L}$-amalgam but no $\ell$-amalgam, we are able to give a negative answer to Problem 1.42 of [6]. This question was the original motivation for the paper.

If $G$ is a p.o. group which is the intersection of right orders, then can $G$ be order-embedded in an $\ell$-group with the embedding preserving any existing suprema and infima?

For our goal, we adapt a Pierce's example [5].

Example 6.1 Let $\Omega_{i}=(\mathbb{Z} \times \mathbb{Z})_{i}(i=1,2)$ ordered lexicographically from the right; i.e., $(n, m) \leq(r, s)$ if either $m<s$ or both $m=s$ and $n \leq r$. Let $\Omega=\Omega_{1} \cup \Omega_{2}$ with $\Omega_{1}<\Omega_{2}$. Consider the subgroup $G \leq \mathrm{A}(\Omega)$ generated by the permutations $a, b, c, h$ where:

$$
\begin{aligned}
& a:(n, 2 m+1)_{1} \mapsto(n+1,2 m+1)_{1}, \quad(n, 2 m+1)_{2} \mapsto(n, 2 m+1)_{2}, \\
& (n, 2 m)_{1} \mapsto(n, 2 m)_{1}, \quad(n, 2 m)_{2} \mapsto(n+1,2 m)_{2} \text {; } \\
& b:(n, 2 m+1)_{1} \mapsto(n, 2 m+1)_{1}, \quad(n, 2 m+1)_{2} \mapsto(n+1,2 m+1)_{2}, \\
& (n, 2 m)_{1} \mapsto(n+1,2 m)_{1}, \quad(n, 2 m)_{2} \mapsto(n, 2 m)_{2} ; \\
& c:(n, m)_{i} \mapsto(n, m+1)_{i} \quad(i=1,2) ; \\
& h:(n, m)_{1} \mapsto(n, m+2)_{1}, \quad(n, m)_{2} \mapsto(n, m)_{2} ; \\
& (n, m)_{i} \in(\mathbb{Z} \times \mathbb{Z})_{i} \quad(i=1,2) .
\end{aligned}
$$

It is easy to see that the subgroup $A=\langle a, b, h\rangle$ is a free abelian group of rank three and that $G$ is the split extension $A \rtimes\langle c\rangle$ of $A$ under the cyclic subgroup with generator the automorphism $c$ given by:

$$
a^{c}=b, \quad b^{c}=a, \quad h^{c}=h .
$$

So $G$ is metabelian; it has 6 defining relations, namely (13) and

$$
[a, b]=[a, h]=[b, h]=1 .
$$

So the elements $g \in G$ have unique canonical form:

$$
g=a^{t_{1}} b^{t_{2}} h^{t_{3}} c^{t_{4}} \quad\left(t_{1}, \ldots, t_{4} \in \mathbb{Z}\right) .
$$


Let $\mathcal{F}=\left\{\prec_{\lambda} \mid \lambda \in \Lambda\right\}$ be the family of all well-orderings of $\Omega$ and $\lambda \in \Lambda$. Let $\leq_{\lambda}$ be the right order on $G$ associated with $\prec_{\lambda} \in \mathcal{F}$; i.e., $1 \leq_{\lambda} g$ if and only if $(n, m)_{i} \leq(n, m)_{i} g$ in $\Omega$, where $(n, m)_{i}$ is the least element of $\operatorname{supp}(g)$ under $\prec_{\lambda}$. Let $\leq_{0}$ be the intersection of all $\leq_{\lambda}(\lambda \in \Lambda)$ and their conjugates.

We claim that $G$ equipped with the partial order $\leq_{0}$ cannot be orderembedded in any $\ell$-group with the embedding preserving the three infima $c^{2} \wedge a, c^{2} h^{-1} \wedge a$ and $h \wedge a$.

We first establish that $g=a^{t_{1}} b^{t_{2}} h^{t_{3}} c^{t_{4}} \geq_{0} 1$ if and only if one of three conditions is fulfilled:

(a) $t_{4}>0$ and $t_{4}+2 t_{3}>0$;

(b) $t_{4}>0, t_{4}+2 t_{3}=0$ and $t_{1}, t_{2} \geq 0$;

(c) $t_{4}=0, t_{3} \geq 0$ and $t_{1}, t_{2} \geq 0$.

If $t_{4}>0$ and $t_{4}+2 t_{3}>0$, then $(n, m)_{1} g=\left(n^{\prime}, m+t_{4}+2 t_{3}\right)_{1}>(n, m)_{1}$ and $(n, m)_{2} g=\left(n^{\prime}, m+t_{4}\right)_{2}>(n, m)_{2}$; so $\alpha g>\alpha$ for all $\alpha \in \Omega$. This gives $1<_{\lambda} g$ for all $\lambda \in \Lambda$. Since conjugates of $g$ have the same exponents $t_{3}, t_{4}$ as $g$, we get $1<_{\lambda} g^{f}$ for all $f \in G$ and $\lambda \in \Lambda$. Hence $1<_{0} g$.

If $t_{4}>0, t_{4}+2 t_{3}=0$ and $t_{1}, t_{2} \geq 0$, then

$$
\begin{gathered}
(n, 2 m+1)_{1} g=\left(n+t_{1}, 2 m+1\right)_{1} \geq(n, 2 m+1)_{1}, \\
(n, 2 m+1)_{2} g=\left(n+t_{2}, 2 m+1+t_{4}\right)_{2}>(n, 2 m+1)_{2}, \\
(n, 2 m)_{1} g=\left(n+t_{2}, 2 m+1\right)_{1} \geq(n, 2 m+1)_{1}, \\
(n, 2 m)_{2} g=\left(n+t_{1}, 2 m+1+t_{4}\right)_{2}>(n, 2 m+1)_{2} .
\end{gathered}
$$

So $\alpha g \geq \alpha$ for all $\alpha \in \Omega$ and $(0,1)_{2} g>(0,1)_{2}$. This gives $1<_{\lambda} g$ for all $\lambda \in \Lambda$. Now $t_{4}$ is even and the conjugates of $g$ are equal to $g$ or to $a^{t_{2}} b^{t_{1}} h^{t_{3}} c^{t_{4}}$, and therefore satisfy condition (b).

If $t_{4}=0, t_{3} \geq 0$ and $t_{1}, t_{2} \geq 0$, then

$$
\begin{gathered}
(n, 2 m+1)_{1} g=\left(n+t_{1}, 2 m+1+2 t_{3}\right)_{1} \geq(n, 2 m+1)_{1}, \\
(n, 2 m+1)_{2} g=\left(n+t_{2}, 2 m+1\right)_{2} \geq(n, 2 m+1)_{2}, \\
(n, 2 m)_{1} g=\left(n+t_{2}, 2 m+1+2 t_{3}\right)_{1} \geq(n, 2 m+1)_{1}, \\
(n, 2 m)_{2} g=\left(n+t_{1}, 2 m+1\right)_{2} \geq(n, 2 m+1)_{2} .
\end{gathered}
$$


Now $\alpha g \geq \alpha$ for all $\alpha \in \Omega$ and if $g \neq 1$, then $\alpha g>\alpha$ for some $\alpha \in \Omega$. So $t_{4}$ is also even and, as above, the conjugates of $g$ are $g$ and $a^{t_{2}} b^{t_{1}} h^{t_{3}} c^{t_{4}}$ and so satisfy condition (c).

In all cases $1 \leq_{0} g$.

Assume now that at least one of the conditions (a) - (c) fails for some $g \in G$. For such an element $g$, we must have either $t_{4}<0$, or $t_{4}+2 t_{3}<0$, or $t_{4}=t_{3}=0$ and one or both of $t_{1}, t_{2}$ is strictly negative. If $t_{4}<0$, then $(0,0)_{2} g<(0,0)_{2}$ and $g \prec_{\lambda} 1$ for the well-ordering $\preceq_{\lambda} \in \mathcal{F}$ with the least point $(0,0)_{2}$. So $1 \nless_{0} g$. If $t_{4}+2 t_{3}<0$, then $(0,0)_{1} g<(0,0)_{1}$, and $1 \nless_{0} g$ similarly. If $t_{4}=t_{3}=0$ and $t_{2}<0$ then $(0,0)_{1} g<(0,0)_{1}$; and if $t_{4}=t_{3}=0$ and $t_{1}<0$ then $(0,0)_{2} g<(0,0)_{2}$. In all cases, $1 \nless_{0} g$.

We next calculate $c^{2} \wedge a, c^{2} h^{-1} \wedge a$ and $h \wedge a$. Using, respectively, conditions (a), (b), (c) and (c), we see that $c^{2}>_{0} 1, c^{2} h^{-1}>_{0} 1, h>_{0} 1$, and $a>_{0} 1$. So all $c^{2} \wedge a, c^{2} h^{-1} \wedge a, h \wedge a$ are positive under $\leq_{0}$. Further, $c^{2} a^{-1}=a^{-1} c^{2}>_{0} 1$ by (a) and so $c^{2} \wedge a=a$. Suppose $g=a^{t_{1}} b^{t_{2}} h^{t_{3}} c^{t_{4}} \in G$ satisfies

$$
\begin{gathered}
1 \leq_{0} g, \\
g \leq_{0} c^{2} h^{-1}, \\
g \leq_{0} a
\end{gathered}
$$

Now (15) and (a) - (c) give $t_{4} \geq 0$ and $t_{4}+2 t_{3} \geq 0$, but (17) and (a) - (c) give $t_{4} \leq 0$ and $t_{4}+2 t_{3} \leq 0$. Thus $t_{4}=t_{3}=0$ and $g=a^{t_{1}} b^{t_{2}}$. Further, (15) and (c) give $t_{1}, t_{2} \geq 0$; but (16) and (c) give $t_{1}, t_{2} \leq 0$. So $g=1$ and consequently $c^{2} h^{-1} \wedge a=1$. Similarly, $h \wedge a=1$.

In any $\ell$-group, $c^{2} h^{-1} \wedge a=1$ and $h \wedge a=1$ imply $c^{2} \wedge a=1$. But we have $c^{2} \wedge a=a$ in the p.o. group $G$. This completes the negative solution of Problem 1.42. //

\section{References}

[1] V. V. Bludov and A. M. W.Glass, Word problems, embeddings, and free products of right-ordered groups with amalgamated subgroup, Proc. London Math. Soc. 2009; doi: 10.1112/plms/pdp008 
[2] A. M. W. Glass, Partially Ordered Groups, Series in Algebra 7, World Scientific Pub. Co., Singapore, 1999.

[3] W. C. Holland, The lattice-ordered group of automorphisms of an ordered set, Michigan Math. J., 10 (1963), 399-408.

[4] W. C. Holland and E. B. Scrimger, Free products of lattice-ordered groups, Algebra Universalis 2 (1972), 247-254.

[5] K. R. Pierce, Amalgamations of lattice-ordered groups, Trans. Amer. Math. Soc. 172 (1972), 249-260.

[6] V. M. Kopytov and N. Ya. Medvedev, Ordered groups, Selected Problems in Algebra. Collection of works dedicated to the memory of N. Ya. Medvedev, Altaii State University, Barnaul, 2007, 15-112 (in Russian).

[7] A. A. Vinogradov, On the free product of ordered groups, Math. Sbornik 25 (1949), 163-168 (in Russian).

Authors' addresses:

V. V. Bludov:

Department of Mathematics, Physics, and Informatics,

Irkutsk State Teachers Training University, Irkutsk 664011,

Russia

vasily-bludov@yandex.ru
A. M. W. Glass:

Queens' College, Cambridge CB3 9ET, England and

Department of Pure Mathematics and Mathematical Statistics, Centre for Mathematical Sciences, Wilberforce Rd., Cambridge CB3 0WB, England amwg@dpmms.cam.ac.uk 